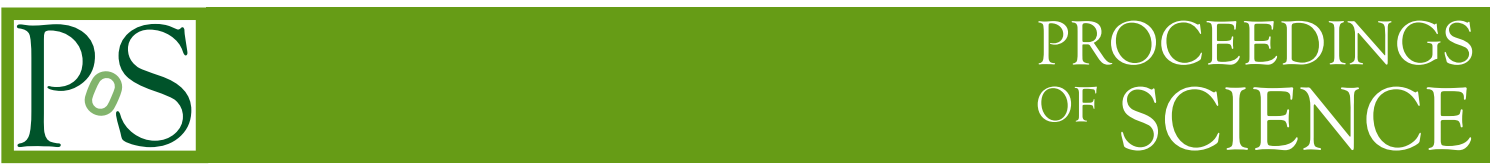

\title{
The BSUIN project
}

National Center for Nuclear Research, Astrophysics Division in Lodz, Poland

E-mail: kj@zpk.u.lodz.pl

K. Jedrzejczak

National Center for Nuclear Research, Astrophysics Division in Lodz, Poland

E-mail: kj@zpk.u.lodz.pl*

Z. Debicki, M. Kasztelan, W. Marszal, J. Orzechowski, M. Przybylak, P. Tokarski

National Center for Nuclear Research, Astrophysics Division in Lodz, Poland

P. Jalas, V. Isoherranen, J. Joutsenvaara, E-R. Niinikoski, R. Heikkila,

University of Oulu

H. Ahola, P. Aro, T. Vuorela

Oulu University of Applied Sciences, Oulu, Finland

J. Kisiel, K. Karpa, K. Szkilniarz

University of Silesia, Katowice, Poland

M. Laaksoharju, M. Ohlsson

Swedish Nuclear Fuel and Waste Management Co., Stockholm, Sweden.

W. Pytel

KGHM S.A., Research development center Cuprum, Wroclaw, Poland

D. Horner, H.Mischo

TU Bergakademie Freiberg, Freiberg, Germany

R. Giese, K. Jaksch

German Research Centre for Geosciences, Potsdam, Germany

V. Mockus, T. Valys

Vilnius University, Vilnius, Lithuania

V. Gostilo

Baltic Scientific instruments, Riga, Latvia

V. Shekov

Karelian Research Center of Russian Academy of Sciences, Petrozavodsk, Russia

A. Stepanov

Khlopin Institute, St Petersburg, Russia

V. Karu, A.Paat

Tallinn University of Technology, Department of Geology, Tallinn, Estonia

(c) Copyright owned by the author(s) under the terms of the Creative Commons

Attribution-NonCommercial-NoDerivatives 4.0 International License (CC BY-NC-ND 4.0).

http://pos.sissa.it/ 
Baltic Sea Underground Innovation Network (BSUIN) is an European Union funded project that extends capabilities of underground laboratories. The aim of the project is to join efforts in making the underground laboratories in the Baltic Sea Region's more accessible for innovation, business development and science by improving the availability of information about the underground facilities, service offerings, user experience, safety and marketing. The development of standards for the characterization of underground laboratories will allow to compared them with each other. This will help you choose the best places for physical measurements such as neutrino physics or searching for dark matter. The project concerns laboratories where so far no measurements have been made, and even undergrounds where there are no organized laboratories yet.The description of the BSUIN project and the first results of characterization of natural radioactive background in underground laboratories will be presentedThe BSUIN Project is funded by Interreg Baltic Sea funding cooperation [2].

36th International Cosmic Ray Conference -ICRC2019-

July 24th - August 1st, 2019

Madison, WI, U.S.A.

${ }^{*}$ Speaker. 


\section{Introduction}

The purpose of the BSUIN project is to develop a service offering of Baltic Sea Region's (BSR) underground laboratories (UL) in order to develop their capability to offer technology transfer utilizing the facilities and research infrastructures of the ULs for business development. Currently the Baltic Sea ULs operate separately and their capacity to offer services to businesses is limited mostly to regional or national level. The project aims to develop the capabilities of ULs in order to improve their service offering as a capacity for innovation and to create a network of the Baltic Sea Region's Uls As a main outcome the BSUIN consortium will create a sustainable network organization, which will disseminate the technical, marketing, operational quality, training and other information about the BSR ULs created during the project.

As a main outcome of the project, the BSUIN consortium creates a sustainable network which enables to disseminate the technical, marketing, operational quality, training and other information about underground laboratories in the Baltic Sea region. The web based tools and the network organization shall work as an open innovation platform for further quality development, innovation and best practices exchange. Project duration is 36 months from October 2017 until September 2020. The total budget is 3.4 MEuro.

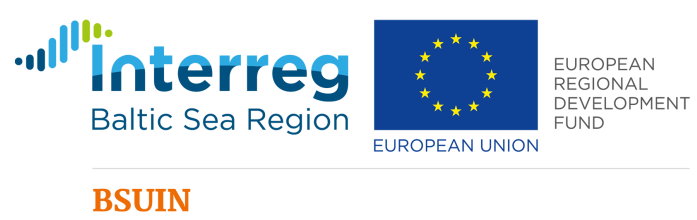

Figure 1: The BSUIN logo.[?]

\section{BSUIN network}

Thirteen BSIUN partners from nine countries around the Baltic Sea are members of the BSUIN consortium. There are two partners from Finland, Germany and Russia, three partners from Poland and a partner from Sweden, Estonia, Latvia and Lithuania in the BSUIN consortium.

List of BSUIN partners: University of Oulu, Kerttu Saalasti Institute (Finland) Oulu University of Applied Sciences (Finland) University of Silesia in Katowice (Poland) Swedish Nuclear Fuel and Waste Management Co (Sweden) KGHM Cuprum Research \& Development Centre Ltd. (Poland) TU Bergakademia Freiberg Technical University (Germany) German Research Centre for Geosciences (Germany) Vilnius University (Lithuania) National Center for Nuclear Research (Poland) Baltic ScientificInstruments (Latvia) Karelian Research Center of Russian Academy of Sciences (Russia) Joint stock company "Khlopin Radium Institute" (Russia) Tallinn University of Technology (Estonia)

There are also eighteen associated partners, who support BSUIN: The Henryk Niewodniczanski Institute of Nuclear Physics Polish Academy of Sciences (Poland), Rockplan Oy (Finland) 
Normet Oy (Finland) K+S GmbH (Germany), Kalmar regional Council (Sweden), Pyhajarvi Municipality (Finland), DMT GmbH (Germany), M-Solutions Oy (Finland), Muon Solutions Oy (Finland), University of Tartu, Institute of Physics (Estonia) Kolmas Karelia LLC (Russia), Geological Institute of Karelia (Russia), Pyhasalmi Mine Oy (Finland) University of Aarhus (Denmark) University of Oulu, Department of Architecture (Finland) University of Jyvaskyla, Department of Physics (Finland), Amberg Group including Versuchsstollen Hagerbach (VSH) (Switzerland), Glowny Instytut Gornictwa (Poland).

There are six, existing or potential, underground laboratories involved in the BSUIN project. They are located in five countries (Finland, Sweden, Germany, Poland, Russia), the exact location is shown in figure 2 .

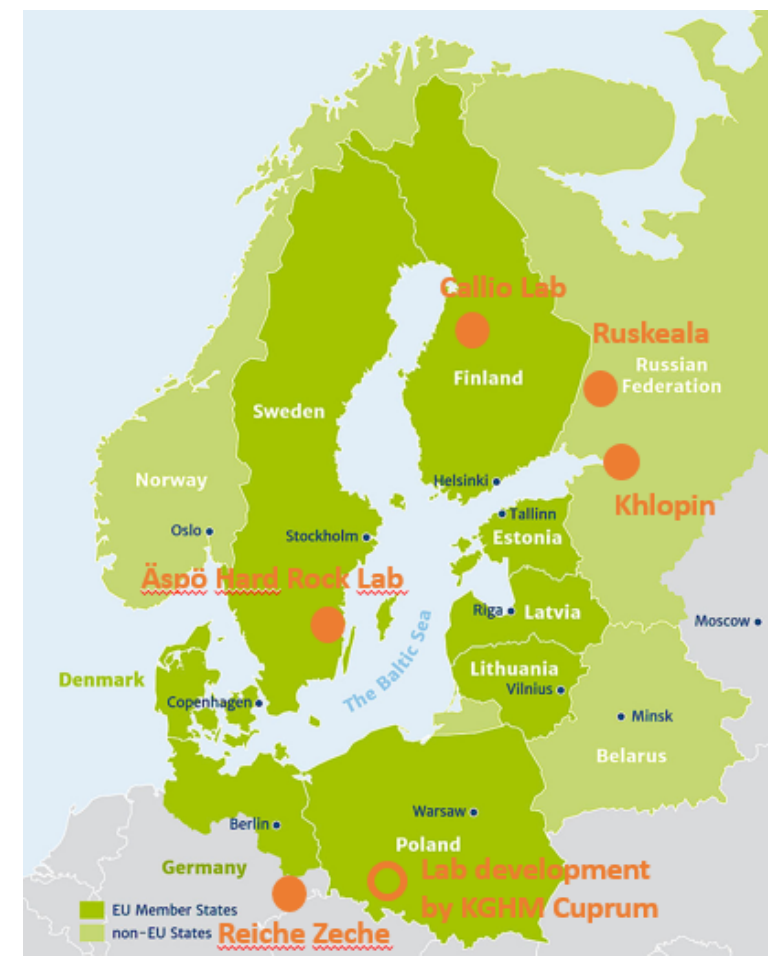

Figure 2: The map of underground laboratories involved in the BSUIN project. Underground laboratories are marked with circles. The INTERREG Baltic Sea support area is also marked. [?]

\section{BSUIN structure}

Work at BSUIN is organized in five Work Packages (WP): WP1 - "Project management and administration", WP2 - "Characterization of underground facilities", WP3 - "Service design, market design and branding of ULs as a capacity for innovation", WP4 - "Underground environment improvement", and WP5 - "Networking of BSR ULs and their users".

Every Work Package is focusing on different aspects of the project.

The main objective of WP2 is characterization of underground locations. Due to the fact that underground locations are significantly different from the Earth's surface, it is necessary to 
characterize individual locations. The characteristics of underground locations presented in an friendly way can be used by potential commercial customers.

The goal of WP3 is to highlight the innovative possibilities that can be developed. Many of the underground locations do not have adequate infrastructure to conduct the organized activities. In order for innovative projects to be developed there, it is necessary to organize safety procedures. Development of standards for the transfer of innovative technologies to the wide application market.

The WP4 package will define the conditions of work organization in ULs and safety rules as well as risk assessment. Dissemination of best practices and development guidelines will be shared.

The last WP5 package is organizing cooperation between underground locations. Creation of a common web page platform for innovative activities and sharing of various types of information, training, security procedures, marketing materials and all kinds of other information that may be useful for other partners.

The structure of BSUIN project and relations between WPs are schematically shown in the figure 3

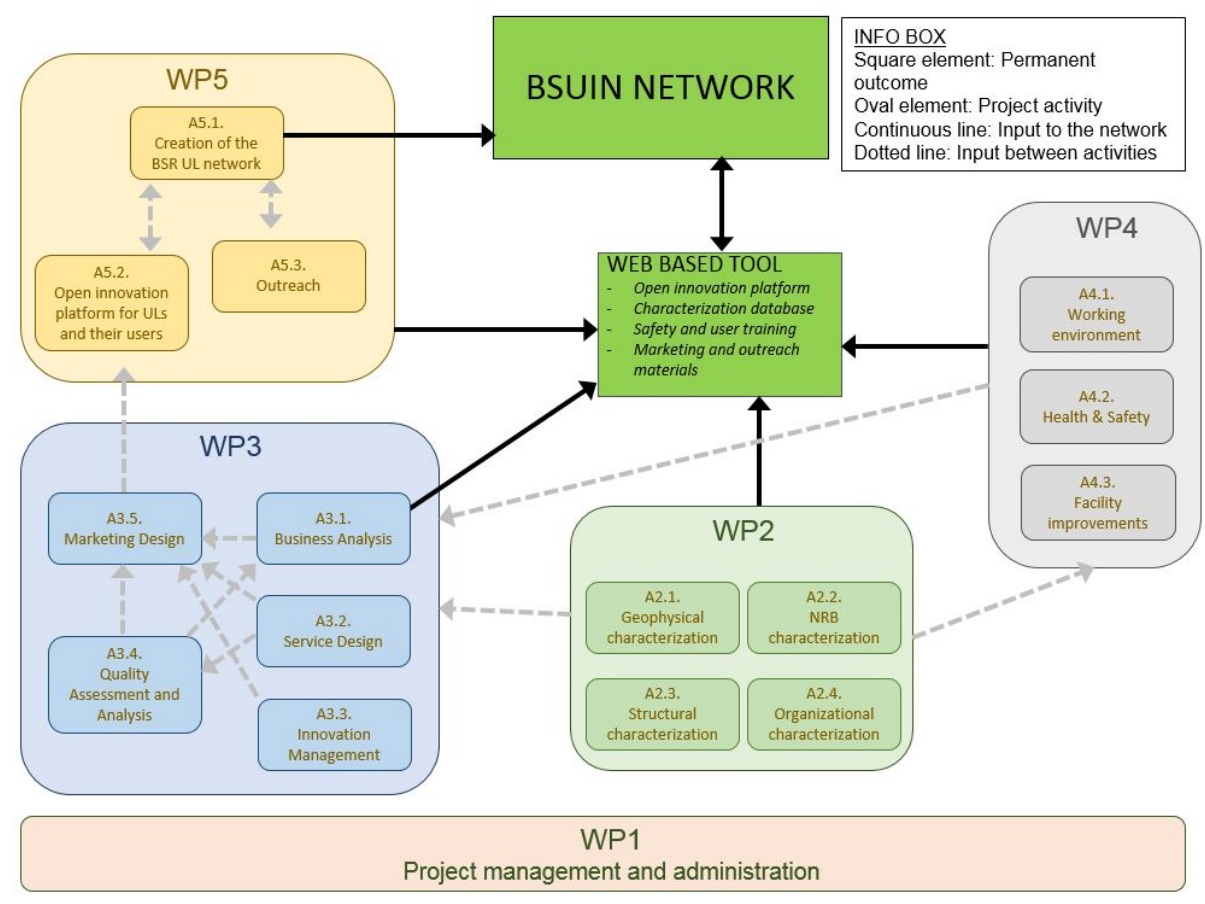

Figure 3: The BSUIN project work packages (WP) scheme. Schematically shown relations between different work packages and the activities. In each of the work packages, institutions from many countries of the Baltic Sea region participate.[?]

\section{WP2: Characterization of underground facilities}

Tasks implemented in Work Package 2 are related to measurements of physical values. WP2 the package is divided into four activities: WP2.1 Geophysical characterization WP2.2 Natural 
radioactive background characterization WP2.3 Structural characterization WP2.4 Organizational characterization The National Centre for Nuclear Research (the first author affiliation) is the leader of activity WP2.2. The goal of this activity is the description of natural radioactivity background (NRB) in BSUIN underground locations. The extremely accurate characterization of NRB is essential in order to enable the utilization of the ULs as low NRB environments for product R\&D, such as development of nuclear measurement or production of materials for nuclear industry or applied scientific research.

An interesting type of NRB is the natural neutron background. Neutrons can be a dangerous background for possible experiments measuring neutrino interactions or seeking dark matter particles or double beta decay without neutrino. All those experiments are waiting for signals appearing in the detector ,of nowhere "ie. when no particles entering the detector is recorded. Neutrons, thanks to their ability of penetration may go undetected into the interior of the detectors there and give a signal imitating the sought effect. That is why so, is important to know their stream in a laboratory environment.

\section{WP2 pilot measurements: minimal setup for thermal neutron flux measurements}

The best method for measuring of the thermal neutron flux is to use helium counters. Helium counters are gas proportional counter filled with helium-3, a helium isotope with large cross-section for thermal neutron capture. In the capture reaction

$$
{ }^{3} \mathrm{He}+n \rightarrow{ }^{3} \mathrm{H}+\mathrm{p}+764 \mathrm{keV}
$$

$764 \mathrm{keV}$ of energy is released and charged particles are produced. Finally, these products are detected by the helium counter in the same way as the standard proportional counter, and characteristic peak $764 \mathrm{keV}$ appears on the energy spectrum. Unfortunately, helium-3 is an artificially isotope and now is very difficult to acquire because all production is intended for safety purposes. This is the reason that we decided to test the simplest setup for thermal flux measurements should be, with the minimum possible number of counters. It is obvious that a setup containing many counters and sophisticated measuring electronics will gave more reliable results than a smaller and simpler one, but in many cases it will be an overkill: unnecessarily raising the complexity and cost of the measurement. We would like to propose a setup as simple as possible, but still able to measure thermal neutron flux in underground laboratories with sufficient reliability.

In our opinion, the setup should have the following features:

- consist of at least two detectors, so that the measurement uncertainty can be determined by comparing the results

- the method of distinguishing real neutron recording from noise should exist

- the presence of the operator during the measurements should not be necessary, which will make very long-term measurements easier and cheaper

We used the setup of ZDAJ NEM425A50 helium counters. ZDAJ NEM425A50 helium counter is made of a $50 \mathrm{~cm}$ long steel tube with a diameter of $2.5 \mathrm{~cm}$. It is filled with helium 
under pressure ${ }^{3} \mathrm{He}$ at $4 \mathrm{~atm}$. and natural krypton under pressure $0.5 \mathrm{~atm}$. Each helium counter had its own, independent front-end, powered and controlled by main computer via USB cable. The main computer itself was completely remotely controlled over the Internet - this is essential for long-term measurements. Each front-end contained an analogue current amplifier, trigger system, analogue-to-digital converter (ADC) and SAM7 microcontroller. SAM7 controlled the entire device and provided USB connectivity. ADC used 8 bits, so signals were digitized to 256 levels. ADC did not have its own hardware clock, but it was clocked with SAM7 commands. Also the measured data circular buffer was an array in the SAM7 RAM memory. This solution simplifies hardware and makes the size of the buffer and the sampling frequency adjustable, but the maximum sampling rate was relatively small, because it was limited by the speed of the program loop in SAM7. Analogue trigger level was correspond to 29 ADC level. For each trigger level exceeded event, 50 samples oscilloscope like wave-form were collected: 14 samples before the trigger and 36 samples after. Then the data was transmitted to the main computer, tagged with the main computer's time and saved to the disk. Finally, the measuring device was reset. The dead time after single event was about 300 microseconds. In the first version of the front-end (measurements in Freiberg) samples were taken every $700 \mathrm{~ns}$ (maximum available counting rate), in the next one sampling rate has been reduced to single sample per $1000 \mathrm{~ns}$, to simplify SAM7 firmware. The $1 \mathrm{MHz}$ sampling rate is sufficient to the pulse-shape analyzes.

Shape analysis relies on differences in the signal rise time, generally the rise time is shorter for noise signal than for neutron signal. The cut is still being developed, good results give full rise time, as well as its largest derivative. An exemplary analysis is shown in the figure 4.

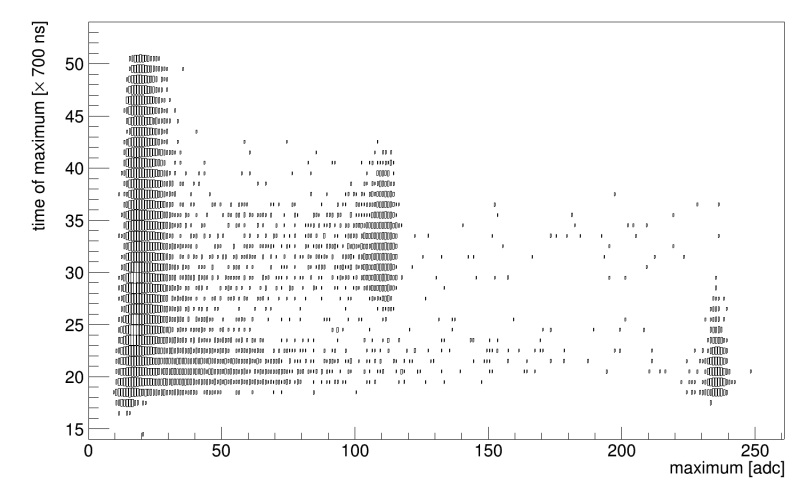

Figure 4: Time of signal maximum (rise time) versus signal maximum. $764 \mathrm{keV}$ peak is clearly visible as a spot with center about 35 unit of rise time and 120 ADC.

We have carried out pilot measurements in three locations, with different configurations of the measurement setup:

- Freiberg (Germany), March - July 2018. Two counters, the first version of front-ends, in the first phase measurement without covers, in the second with a borax shield. Remote control tests. 
- Pyhasalmi (Finland) at the turn of July and August 2018. 10 meters, the second version of front-ends. Changed way of connecting high voltage, added the ability to remotely change software in microcontrollers, a new control program on a PC. Series of short (days) measurements in various configurations, with polyethylene covers and water (in plastic canisters).

- "Barbara" experimental mine (Poland) 14 February 2019. and they last (see figure 5). 5 counters without cover and 5 with polyethylene cover. Improved version of Pyhasalmi frontends. All noises have been removed. New software on microcontrollers to facilitate the launch of the apparatus after a remote reboot. Improved control program on the PC

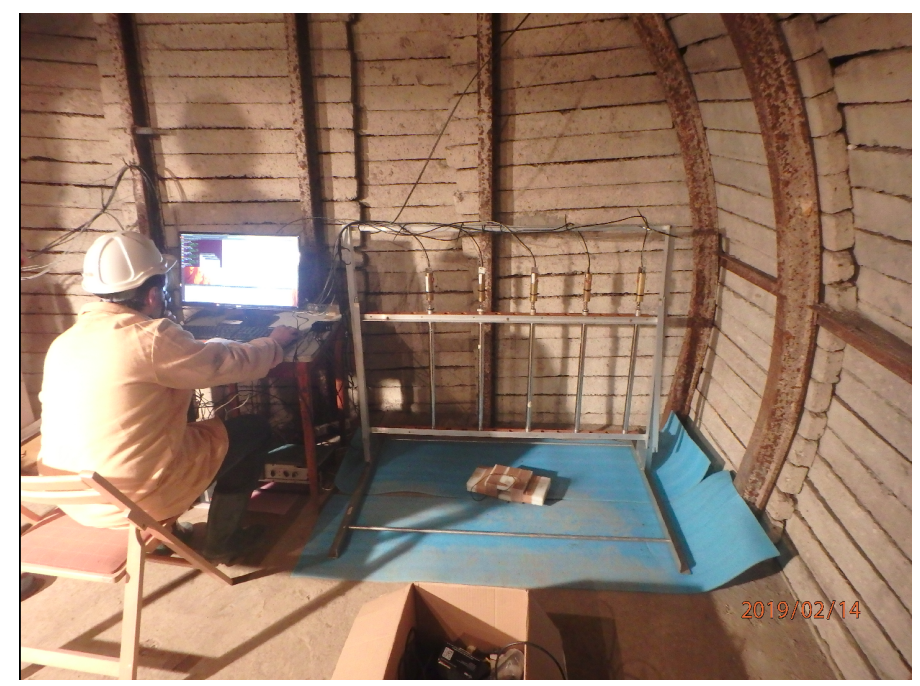

Figure 5: Measurements in the "Barbara" experimental mine. Five helium counters without a polyethylene cover are visible.

Further measurements are planned.

This work is supported by European Regional Development Fund of European Union and is a part of „Baltic Sea Underground Innovation Network” (BSUIN) Project. The BSUIN Project is funded by Interreg Baltic Sea funding cooperation [2].

This work is supported by Polish Ministry of Science and Higher Education.

\section{References}

[1] The BSUIN project web page https://bsuin.eu

[2] The BSUIN project on INTERREG Baltic Sea web page https://projects.interreg-baltic.eu/projects/bsuin-102.html

[3] Debicki, Z, Jedrzejczak, K, Kasztelan, M, Marszal, W, Orzechowski, J, Szabelski, J, Tokarski, P. (2019). The BSUIN project - overview and same results. Journal of Physics: Conference Series. 1181. 012071. 10.1088/1742-6596/1181/1/012071. 